\title{
Efficacy of Different Types of Exercise-Based Cardiac Rehabilitation on Coronary Heart Disease: a Network Meta-analysis
}

\author{
Tian-li Xia, Fang-yang Huang, Yong Peng, Bao-tao Huang, Xiao-bo Pu, Yong Yang, Hua Chai, and \\ Mao Chen
}

Department of Cardiology, West China Hospital, Sichuan University, Chengdu, People's Republic of China.

BACKGROUND: Exercise-based cardiac rehabilitation (CR) has been recognized as an essential component of the treatment for coronary heart disease (CHD). Determining the efficacy of modern alternative treatment methods is the key to developing exercise-based CR programs.

METHODS: Studies published through June 6, 2016, were identified using MEDLINE, EMBASE, and the Cochrane Library. English-language articles regarding the efficacy of different modes of $\mathrm{CR}$ in patients with CHD were included in this analysis. Two investigators independently reviewed abstracts and fulltext articles and extracted data from the studies. According to the categories described by prior Cochrane reviews, exercise-based CR was classified into center-based CR, home-based CR, tele-based $\mathrm{CR}$, and combined CR for this analysis. Outcomes included all-cause mortality, cardiovascular death, recurrent fatal and/or nonfatal myocardial infarction, recurrent cardiac artery bypass grafting, recurrent percutaneous coronary intervention (PCI), and hospital readmissions.

RESULTS: Sixty randomized clinical trials ( $n=$ 19,411) were included in the analysis. Network meta-analysis (NMA) demonstrated that only centerbased CR significantly reduced all-cause mortality (center-based: $\mathrm{RR}=0.76$ [95\% CI 0.64-0.90], $p=$ 0.002) compared to usual care. Other modes of CR were not significantly different from usual care with regard to their ability to reduce mortality. Treatment ranking indicated that combined $\mathrm{CR}$ exhibited the highest probability (86.9\%) of being the most effective mode, but this finding was not statistically significant due to the small sample size (combined: $\mathrm{RR}=0.50$ [95\% CI 0.20-1.27], $p=0.146$ ).

CONCLUSIONS: Current evidence suggests that centerbased CR is acceptable for patients with CHD. As homeand tele-based CR can save time, money, effort, and resources and may be preferred by patients, their efficacy should be investigated further in subsequent studies.

Tian-li Xia and Fang-yang Huang contributed equally to this work.

Electronic supplementary material The online version of this article (https://doi.org/10.1007/s11606-018-4636-y) contains supplementary material, which is available to authorized users.

Received April 17, 2017

Revised October 16, 2017

Accepted August 6, 2018

Published online September 13, 2018
KEY WORDS: cardiac rehabilitation; coronary artery disease; telemedicine; meta-analysis.

$\mathrm{J}$ Gen Intern Med 33(12):2201-9

DOI: $10.1007 / \mathrm{s} 11606-018-4636-\mathrm{y}$

๑) Society of General Internal Medicine 2018

\section{INTRODUCTION}

Cardiac rehabilitation (CR) programs have been recognized as an essential component of treatments for coronary heart disease (CHD). ${ }^{1-3} \mathrm{CR}$ programs are medical multidisciplinary programs consisting of exercise and education sessions designed to help patients achieve optimal physical and functional status and prevent further cardiac illness. ${ }^{4} \mathrm{CR}$ programs can improve physical health and contribute to substantial decreases in morbidity and mortality. Therefore, these programs are highly recommended by clinical guidelines. ${ }^{5}$

Traditionally, CR is undertaken in hospital-based settings and in outpatient clinics. Previous studies have established this type of CR as the basic mode of CR. ${ }^{6,7}$ Despite the effectiveness of this mode, several barriers, including low referrals, low uptake, and poor adherence, frequently prevent its widespread use. Since the mid-1980s, home-based exercise programs have served as an alternative to traditional CR for myocardial infarction (MI) patients. ${ }^{8}$ Since the 1990s, the use of homebased CR among low-risk CHD patients has become more widespread. However, physicians prefer using center-based CR programs over home-based CR programs in patients with more severe diseases or patients experiencing complications from their diseases. To ensure the provision of safe and effective care for these patients, physicians have attempted to monitor their vital signs using portable heart rate monitors since the mid-1980s. ${ }^{9}$ The development and progression of sensor technology, communication technology, and data analysis techniques over the past 20 years have spurred the creation of modified home-based modes combining the accessibility of home-based CR with the specialist monitoring and support services of center-based CR. ${ }^{10}$ This mode of CR, known as tele-based CR, is expected to be a safe means of avoiding inappropriate exercise while facilitating widespread patient participation in CR programs. However, as the findings of previous studies regarding this issue are inconsistent, whether these modern modes are viable alternatives to 
center-based CR problems remains unknown. ${ }^{11,}{ }^{12}$ Given the few head-to-head trials evaluating the efficacy of multiple interventions, we conducted a network meta-analysis (NMA) to comprehensively compare and rank the efficacy of centerbased, home-based, and tele-based CR in patients with CHD.

\section{METHODS}

Studies published through June 6, 2016, were systematically searched in MEDLINE (through Ovid SP), EMBASE (through Ovid SP), and CENTRAL (through the Cochrane Library). The full study protocol, including a detailed research strategy, was registered with PROSPERO (ID CRD42016035472). The references of relevant studies and review articles were also checked to identify additional studies.

The inclusion criteria for this analysis were randomized clinical trials (RCTs) of adult patients $(\geq 18$ years) with CHD that compared different types of exercise-based CRs or compared exercise-based CRs with usual care as a component of the treatment. Studies were excluded if CR was performed on all patients to compare the efficacy of different training periods. RCTs that measured the effect of counseling methods but not of exercise training were excluded from the analysis, as were conference abstracts, reviews (including systematic reviews), editorials, observational studies, animal experiments, and pediatric trials. The articles were screened in accordance with the above inclusion and exclusion criteria. Additionally, a standardized data extraction form was designed to collect information regarding the first author, publication year, method, setting, and follow-up duration as well as information regarding the total numbers of patients, the participant characteristics, and the main results of the eligible trials. The data were independently extracted by authors who read the full texts of each article.

For the purpose of this review, exercise-based CR was defined as center-based, home-based, tele-based, and combined CR interventions. Center-based CR was defined as CR undertaken in a hospital or a center with equivalent infrastructure. Home-based programs were defined as those undertaken in patients' homes or in places other than hospitals, such as places in the community, using traditional follow-up methods (e.g., telephone conversations or regular visits). Tele-based CR was defined as CR undertaken in patients' homes or in places other than hospitals that was monitored and guided by health professionals using telehealth technologies. Combined $\mathrm{CR}$ was defined as either (1) both center-based and homebased CR or (2) both center-based and tele-based CR.

The primary endpoint of the analysis was all-cause death. Secondary endpoints were cardiovascular death (CV death), recurrent fatal and/or nonfatal MI, recurrent coronary artery bypass grafting (CABG), recurrent percutaneous coronary intervention (rePCI), and hospital readmission during follow-up.
The methodological quality of the studies was evaluated by two reviewers (FY.H. and Y.P.) using the Cochrane Collaboration's recommended tool for assessing the risk of bias. ${ }^{13}$

We defined low overall risk of bias as follows: low risk in the domains of randomization, outcome assessor blinding, incomplete outcome data, and selective reporting and no other domains with a high risk. Unclear overall risk of bias was defined as at least one unclear risk of bias in the domains of randomization, blinding, incomplete outcome data, and selective reporting and no other domains with a high risk. High overall risk of bias was defined as a high risk of bias in at least one of the following domains: randomization, outcome assessor blinding, incomplete outcome data, or selective reporting.

Pairwise meta-analyses were first performed to estimate the available direct relative effects of the competing interventions using the random effects model in the metan package of Stata 13.0. ${ }^{14}$ In these analyses, we estimated heterogeneity parameters for each pairwise comparison. ${ }^{15,16}$ Subsequently, we conducted a random effects model NMA for each outcome using the multivariate meta-analysis approach in the network package (version 1.2.0) of Stata 13.0. ${ }^{17}$ Network consistency was evaluated by using the loop-specific approach (assuming common heterogeneity across all loops in all-cause death) and comparing the direct estimates with the indirect estimates for each comparison, with a node splitting technique in which the summary estimate of each node is separated into its direct and indirect components. ${ }^{18,}{ }^{19}$ Subgroup NMA was also performed. Older patients (older than 70 years old), patients diagnosed with acute myocardial infarction (AMI), and the publication year of the articles were included as prespecified subgroups. Surface under the cumulative ranking (SUCRA) for each intervention was calculated from a cumulative ranking probability that an intervention was above a certain ranking, taking values between 0 (certainly the worst intervention) and 1 (certainly the best intervention). ${ }^{20}$ The hierarchy of the competing treatment outcomes was determined by calculating the SUCRA for the total population and all subgroups to determine the probability that a particular treatment would be among the best treatments. We performed four sensitivity analyses for the primary outcomes and other secondary outcomes available: (1) we included only studies with a low risk of overall bias; (2) we excluded studies with high overall risk; and (3) we excluded the study published by the WHO in $1984,{ }^{21}$ which had the greatest weight in the analysis and, thus, may have driven the findings.

\section{RESULTS}

The searches identified 13,675 potentially relevant articles. We excluded 13,615 citations that did not meet the eligibility criteria. After full-text screening, we ultimately included 60 RCTs $(n=19,411)$ conducted between 1981 and 2016 (see Appendix 1). Table 1 presents the characteristics of all included studies and study settings. ${ }^{4,9,21-78}$ As indicated in the 
Table 1 Study Characteristics of the Included Trials

\begin{tabular}{|c|c|c|c|c|c|}
\hline$\overline{\text { Study }}$ & Method & Country & $\begin{array}{l}\text { Length of } \\
\text { follow-up (month) }\end{array}$ & Sample size & $\begin{array}{l}\text { Intervention (center-based: } 1 \text {; } \\
\text { home-based: } 2 \text {; tele-based: } 3 \text {; c } \\
\text { ombined: } 4 \text {; control: } 0 \text { ) }\end{array}$ \\
\hline Aronov $2010^{22}$ & Multicenter & Russia & 12 & 392 & 1 vs. 0 \\
\hline Belardinelli $2001^{23}$ & Single center & Italy & 33 & 118 & 1 vs. 0 \\
\hline Bell $1998^{24}$ & Multicenter & Italy & 12 & 252 & 1 vs. 2 \\
\hline Bengtsson $1983^{26}$ & Single center & Sweden & 14 & 171 & 1 vs. 0 \\
\hline Bertie $1992^{27}$ & Single center & UK & 24 & 110 & 1 vs. 0 \\
\hline Bethell $1990^{25}$ & Single center & UK & 60 & 229 & 1 vs. 0 \\
\hline Briffa $2005^{28}$ & Multicenter & Australia & 24 & 113 & 1 vs. 0 \\
\hline Carlsson $1998^{29}$ & Single center & Sweden & 12 & 235 & 1 vs. 4 \\
\hline Carson $1982^{30}$ & Single center & UK & 36 & 303 & 1 vs. 0 \\
\hline COACH pilot $2002^{31}$ & Single center & Australia & 6 & 245 & 3 vs. 0 \\
\hline $\mathrm{COACH}$ trial $2003^{32}$ & Multicenter & Australia & 6 & 792 & 3 vs. 0 \\
\hline Dalal $2007^{33}$ & Single center & UK & 9 & 104 & 1 vs. 2 \\
\hline DeBusk $1994^{34}$ & Multicenter & USA & 12 & 585 & 3 vs. 0 \\
\hline Dugmore $1999^{35}$ & Single center & UK & 60 & 124 & 1 vs. 0 \\
\hline Engblom $1996^{4}$ & Single center & Finland & 60 & 228 & 1 vs. 0 \\
\hline Erdman $1986^{36}$ & Single center & Netherlands & 60 & 80 & 1 vs. 2 \\
\hline Fletcher $1994^{37}$ & Single center & USA & 6 & 88 & 2 vs. 0 \\
\hline Fridlund $1991^{38}$ & Single center & Sweden & 60 & 178 & 1 vs. 0 \\
\hline Giallauria $2009^{40}$ & Single center & Finland & 60 & 61 & 1 vs. 0 \\
\hline Gordon $2002^{39}$ & NR & USA & 30 & 155 & 1 vs. 2 \\
\hline Haskell $1994^{41}$ & Multicenter & USA & 48 & 300 & 2 vs. 0 \\
\hline Heller $1993^{42}$ & Multicenter & Australia & 6 & 450 & 2 vs. 0 \\
\hline Higgins $2001^{43}$ & Single center & Australia & 51 & 105 & 2 vs. 0 \\
\hline Hofman-Bang $1999^{44}$ & Single center & Sweden & 24 & 87 & 1 vs. 0 \\
\hline Houle $2012^{45}$ & Multicenter & Canada & 12 & 65 & 2 vs. 0 \\
\hline Jolly $2007^{46}$ & Multicenter & UK & 24 & 525 & 1 vs. 2 \\
\hline Jones $1996^{47}$ & Multicenter & UK & 12 & 2328 & 1 vs. 0 \\
\hline Kallio $1979^{48}$ & Multicenter & Finland & 36 & 375 & 1 vs. 0 \\
\hline Kovoor $2006^{49}$ & Multicenter & Australia & 6 & 142 & 2 vs. 0 \\
\hline La Rovere $2002^{50}$ & Single center & Italy & 120 & 95 & 1 vs. 0 \\
\hline Lear $2003^{51}$ & NR & Canada & 12 & 302 & 3 vs. 0 \\
\hline Lewin $1992^{52}$ & Single center & Scotland, UK & 12 & 176 & 2 vs. 0 \\
\hline Maddison $2015^{53}$ & Multicenter & New Zealand & 6 & 171 & 2 vs. 0 \\
\hline Manchanda $2000^{54}$ & Single center & India & 12 & 42 & 4 vs. 0 \\
\hline Maroto $2005^{55}$ & Single center & Spain & 120 & 180 & 1 vs. 0 \\
\hline Miller $1984^{9}$ & NR & USA & 6 & 198 & 1,4 vs. 0 \\
\hline Munk $2009^{56}$ & Single center & Norway & 6 & 40 & 1 vs. 0 \\
\hline Mutwalli $2012^{57}$ & Single center & Kingdom of Saudi Arabia & 6 & 49 & 2 vs. 0 \\
\hline Niebauer $1997^{58}$ & NR & UK & 3 & 113 & 2 vs. 0 \\
\hline Oerkild $2012^{59}$ & Single center & Denmark & 12 & 40 & 2 vs. 0 \\
\hline Oldridge $1991^{60}$ & Multicenter & Canada & 12 & 201 & 1 vs. 0 \\
\hline Ornish $1998^{61}$ & Multicenter & USA & 60 & 48 & 1 vs. 0 \\
\hline Reid $2012^{62}$ & Multicenter & Canada & 12 & 223 & 3 vs. 0 \\
\hline Roman $1983^{63}$ & Single center & Chile & 108 & 193 & 1 vs. 0 \\
\hline Schuler $1992^{64}$ & Single center & Germany & 72 & 113 & 2 vs. 0 \\
\hline Seki $2008^{65}$ & Single center & Japan & $\mathrm{NR}$ & 39 & 4 vs. 0 \\
\hline Shaw $1981^{66}$ & Multicenter & USA & 60 & 651 & 1 vs. 0 \\
\hline Sivarajan $1982^{67}$ & Single center & USA & 6 & 258 & 1 vs. 0 \\
\hline Specchia $1996^{68}$ & Single center & Italy & 40 & 256 & 1 vs. 0 \\
\hline Stern $1983^{69}$ & Multicenter & USA & 36 & 106 & 1 vs. 0 \\
\hline Toobert $2000^{70}$ & NR & USA & 12 & 25 & 1 vs. 0 \\
\hline Vermeulen $1983^{71}$ & NR & Netherlands & 60 & 98 & 1 vs. 0 \\
\hline VHSG $2003^{72}$ & Single center & Norway & 60 & 197 & 1 vs. 0 \\
\hline Wang $2012^{73}$ & Multicenter & China & 6 & 160 & 2 vs. 0 \\
\hline West $2012^{74}$ & Multicenter & UK & 24 & 1813 & 1 vs. 0 \\
\hline WHO $1984^{21}$ & Multicenter & European countries & 36 & 3184 & 1 vs. 0 \\
\hline Wilhelmsen $1975^{75}$ & Single center & Sweden & 60 & 315 & 1 vs. 0 \\
\hline Young $2003^{77}$ & Single center & Canada & 12 & 146 & 2 vs. 0 \\
\hline $\mathrm{Yu} 2004^{76}$ & Single center & China & 24 & 269 & 4 vs. 0 \\
\hline Zwisler $2008^{78}$ & Single center & Denmark & 12 & 770 & 1 vs. 0 \\
\hline
\end{tabular}

$N R$, not reported; AMI, acute myocardial infarction

network plot (Fig. 1), center-based CR versus usual care was the most prevalent comparison.

The intensity and types of exercise, frequency and lengths of sessions, and duration of rehabilitation are the keystones of CR programs. We describe each of these factors in detail in Appendix 2; however, only half of the studies reported information regarding these variables. Regarding exercise type, 11 out of 37 trials evaluated center-based CR programs comprising aerobic exercises and strength training, while the others evaluated programs comprising only aerobic exercises. Sessions ranged from $20 \mathrm{~min}$ to $2 \mathrm{~h}$ in length in individual CR programs. There were no significant differences in $\mathrm{CR}$ 


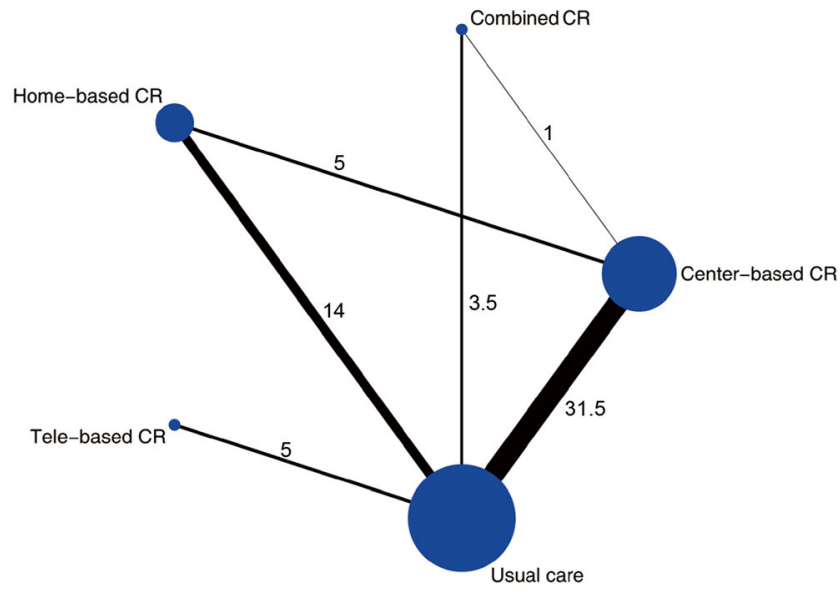

Figure 1 Network of interventional treatments comparing types of cardiac rehabilitation (CR) and usual care. The sizes of the nodes represent the total sample size for each treatment. Line thickness and the numbers beside the lines correspond to the number of trials comparing the interventions. A 3-arm study comparing usual care, center-based CR, and combined CR was included. We considered it as half of a trial.

program duration among the three individual $\mathrm{CR}$ programs (center-based, 6.36 months; home-based, 6.13 months; telebased, 5.70 months; combined, 8.00 months); however, combined CR programs were of significantly longer durations than other programs.

The overall risk of bias of the included RCTs is presented in Figure 2 and Appendix 3. A low risk of random sequence generation was observed in $25(41.7 \%)$ trials, and $13(21.7 \%)$ trials were classified as having a low risk of bias regarding allocation concealment. Fifteen $(25.0 \%)$ trials blinded outcome assessment, 7 (11.7\%) trials did not blind outcome assessment, and the other trials did not report details about blinding. Twenty-six (43.3\%) trials reported incomplete outcome data. Between-group baseline characteristics were balanced in most trials (80.0\%). Intention-to-treat analysis was performed in $24(40.0 \%)$ trials. Twenty-seven (45.0\%) trials evaluated additional treatments beyond exercise-based rehabilitation (e.g., counseling).

Pairwise meta-analyses comparing the $\mathrm{CR}$ programs are presented in Appendix 4. Little heterogeneity was observed in the pairwise comparisons (Appendix 5).

NMA demonstrated that only center-based CR significantly reduced all-cause mortality compared to usual care ( $\mathrm{RR}=$ $0.76,95 \%$ CI $0.64-0.90, p=0.002$ ), while the other types of $\mathrm{CR}$ were not significantly different from usual care with regard to mortality (home-based vs. usual care: $\mathrm{RR}=0.86,95 \% \mathrm{CI}$ $0.55-1.33$; tele-based vs. usual care: $\mathrm{RR}=0.97,95 \% \mathrm{CI} 0.62-$ 1.52 ; combined vs. usual care: $\mathrm{RR}=0.50,95 \% \mathrm{CI} 0.20-1.27$ ). Furthermore, no statistically significant differences were found when comparing different types of CR (center-based vs. home-based CR: $\mathrm{RR}=0.89,95 \%$ CI $0.57-1.40$; centerbased vs. tele-based CR: $\mathrm{RR}=0.78,95 \%$ CI $0.49-1.27$; center-based vs. combined CR: RR $=0.78,95 \%$ CI 0.49 1.27) (Table 2). There was no inconsistency in the NMA estimates (Appendix 4), and there were no significant differences between direct and indirect estimates in closed loops that allowed assessment of network coherence (Appendix 6). The SUCRA value, which indicates the probability that each intervention ranks first, was highest for combined CR (86.9\%), followed by center-based CR (68.4\%), home-based CR (45.7\%), and tele-based CR (29.7\%) (Appendix 7).

NMA demonstrated that center-based CR was significantly associated with a lower incidence of $\mathrm{CV}$ death than usual care (center-based CR vs. usual care: $\mathrm{RR}=0.65,95 \%$ CI 0.49 $0.85, p=0.002$ ), a finding consistent with the standard metaanalysis (Table 2, Appendix 4). Comparison of the efficacy of center-based, home-based, tele-based CR, and combined CR in patients with CHD showed imprecise estimates regarding
Random sequence generation (selection bias)

Allocation concealment (selection bias)

Blinding (detection bias)

Incomplete outcome data (attrition bias)

Selective reporting (reporting bias)

Groups balanced at baseline

Intention-to-treat analysis conducted

口Low risk
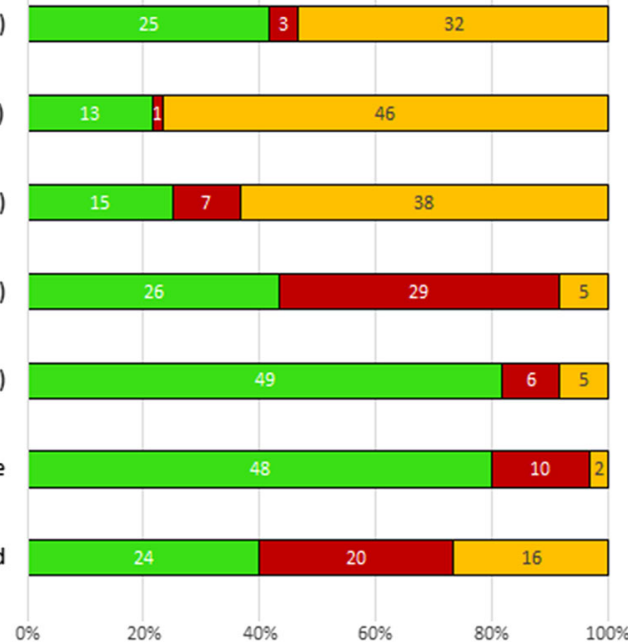

\begin{tabular}{l|l|l}
13 & 1 & 46
\end{tabular}

$26 \quad 29$

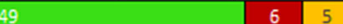

\begin{tabular}{l|l}
\hline 48 & 10
\end{tabular}

$\square$ Unclear risk

Figure 2 Risk of bias graph for the included studies. The figure displays the numbers (and percentages along the $x$-axis) of studies that had low, high, or unclear risk of bias for each item. 
Table 2 Relative Risk and 95\% CIs for the Network Meta-analysis Comparing Every Pair of the 5 Interventions Regarding All-Cause Death (Upper Triangle) and the Column-Defining Treatment Regarding CV Death (Lower Triangle)

\begin{tabular}{llllll}
\hline \hline & Center-based & Home-based & Tele-based & Combined & Control \\
\hline Center-based & NA & $0.89(0.57-1.40)$ & $0.78(0.49-1.27)$ & $0.78(0.49-1.27)$ & $0.76^{*}(0.64-0.90)$ \\
Home-based & $0.41(0.06-2.84)$ & NA & $0.88(0.47-1.65)$ & $0.88(0.47-1.65)$ & $0.86(0.55-1.33)$ \\
Tele-based & $1.34(0.56-3.23)$ & $3.29(0.41-26.68)$ & NA & $0.52(0.19-1.45)$ & $0.97(0.62-1.52)$ \\
Combined & $0.68(0.01-36.71)$ & $1.66(0.02-138.06)$ & $0.51(0.01-29.47)$ & NA & $0.50(0.20-1.27)$ \\
Control & $0.65^{*}(0.49-0.85)$ & $1.58(0.23-10.78)$ & $0.48(0.21-1.11)$ & $0.95(0.02-50.91)$ & NA \\
\hline
\end{tabular}

Comparisons between treatments should be read from left to right, and the relative risk is in the cell intersecting the column-defining treatment and the row-defining treatment. Relative risks less than 1 favor the row-defining treatment regarding all-cause death and the column-defining treatment regarding $C V$ death

$* p=0.002$

CV death, cardiovascular death; NA, not applicable

CV death (center-based vs. home-based CR: $\mathrm{RR}=0.41,95 \%$ CI 0.06-2.84; center-based vs. tele-based CR: $\mathrm{RR}=1.34,95 \%$ CI 0.56-3.23; center-based vs. combined CR: RR $=0.68,95 \%$ CI 0.01-36.71). With respect to preventing AMI, readmission, CABG, or rePCI, no differences were observed when we compared the efficacy of the CR programs with that of usual care. Furthermore, home-based CR, tele-based CR, and combined $\mathrm{CR}$ did not reduce the risks of AMI, readmission, CABG, or rePCI compared with center-based CR (Appendixes 8-9).

To determine whether the efficacy of exercise-based CR was influenced by confounding factors, such as publication time, counseling, age, or diagnosis of AMI, we stratified patients according to these factors (Table 3, Appendix 10). Patients were stratified by the publication time of the article in the first subgroup analysis, which showed that results from the period of 1974-1990 were consistent with those of total patients (center-based CR vs. usual care: $\mathrm{RR}=0.71,95 \% \mathrm{CI}$ $0.58-0.89, p=0.001$ ). Meanwhile, the reported efficacies of other CRs were not statistically different based on publication time, which may have resulted from inadequate statistical power. The second and third subgroup analyses reported that the efficacy of different CRs significantly differs based on the age and type of patients being treated. The type of diagnosis may significantly influence the reported effect of different CRs. The efficacy of CRs in AMI patients and patients younger than 60 years was highly consistent with that in total patients (AMI: center-based CR vs. usual care: $\mathrm{RR}=0.75$, 95\% CI 0.61-0.92, $p=0.001$; younger than 60 years: centerbased CR vs. usual care: $\mathrm{RR}=0.70,95 \%$ CI $0.58-0.84, p=$ 0.001). In other groups, the efficacy of CRs was not significantly different, which may have resulted from inadequate statistical power. The fourth subgroup meta-analysis demonstrated no significant effect based on whether the comparison condition contained counseling (with counseling: $\mathrm{RR}=0.73$, 95\% CI 0.55-0.96, $p=0.024$; without counseling: $\mathrm{RR}=0.77$, $95 \%$ CI $0.61-0.97, p=0.024)$.

Common estimates for heterogeneity variance were low (tau value $\approx 0$ ).

The sensitivity analysis restricted to studies with low overall risk of bias yielded analogous results but showed less power than our primary analysis (center-based: $\mathrm{RR}=0.95,95 \% \mathrm{CI}$ 0.47-1.89) (Appendix 11). Additionally, when we excluded only studies with a high risk of several biases, center-based CR was statistically different from usual care (center-based: $\mathrm{RR}=$ 0.75, 95\% CI 0.58-0.97) (Appendix 12). Results from the analysis without the WHO 1984 paper were consistent with the overall results (center-based: $\mathrm{RR}=0.77,95 \%$ CI $0.63-$ 0.94) (Appendix 13). ${ }^{21}$

\section{DISCUSSION}

This NMA was the first to demonstrate the efficacy of currently available CR strategies for CHD patients. Specifically, this study found strong evidence that the risk of all-cause mortality and $\mathrm{CV}$ mortality was lower in cardiac patients receiving center-based $\mathrm{CR}$ than that in patients receiving usual care. Home-based CR and tele-based CR were not superior to center-based CR and usual care, as the differences between these groups and the usual care group were not statistically significant. In addition, the difference in the risk of AMI, reCABG, rePCI, and hospital readmissions between centerbased CR and usual care was not statistically significant. The efficacy of different CR strategies showed also not significantly different based on the outcomes.

The most recent Cochrane review, which included 14,486 patients with $\mathrm{CHD}$, demonstrated that exercise-based CR exerted important beneficial effects. Specifically, exercise decreased the incidence of $\mathrm{CV}$ mortality and hospital readmission and improved health-related quality of life, findings that are consistent with those of our study. ${ }^{5}$ In our study, we subdivided the exercise-based CR by type to find the most effective approaches for patients. We found that center-based exercise CR resulted in the most benefits to patients. To date, the mainstay approaches to exercise-based CR in many countries depend on using inpatient and outpatient center-based programs, which often take place in supervised settings, such as universities, hospitals, or community locations. Due to several insurmountable barriers associated with center-based $\mathrm{CR}$, including limited program availability, transportation restrictions, and inconvenient program schedules as well as 
Table 3 Subgroup Network Meta-analysis Results of Exercise-Based CR Compared to Usual Care and the Surface Under the Cumulative Ranking Curve (SUCRA)

\begin{tabular}{|c|c|c|c|c|c|c|}
\hline & \multicolumn{2}{|l|}{ Subgroup 1} & \multicolumn{2}{|l|}{ Subgroup 2} & \multicolumn{2}{|l|}{ Subgroup 3} \\
\hline & RR (95\% CI) & SUCRA & RR (95\% CI) & SUCRA & RR (95\% CI) & SUCRA \\
\hline Row 1: publication year & \multicolumn{2}{|l|}{$1974-1990$} & \multicolumn{2}{|l|}{ 1991-2000 } & \multicolumn{2}{|l|}{ 2001-2014 } \\
\hline Usual care & 1 & $7.4 \%$ & 1 & $41.9 \%$ & 1 & $25.1 \%$ \\
\hline Center-based & $0.71(0.58-0.89)$ & $43.9 \%$ & $0.74(0.46-1.18)$ & $77.0 \%$ & $0.88(0.54-1.43)$ & $44.3 \%$ \\
\hline Home-based & $0.07(0.00-1.37)$ & $87.6 \%$ & $1.02(0.53-1.97)$ & $41.9 \%$ & $0.82(0.43-1.57)$ & $51.1 \%$ \\
\hline Tele-based & - & - & $1.20(0.51-2.83)$ & $30.5 \%$ & $0.86(0.44-1.68)$ & $45.7 \%$ \\
\hline Combined & $0.25(0.01-5.15)$ & $61.1 \%$ & $0.78(0.13-4.74)$ & $58.7 \%$ & $0.46(0.14-1.50)$ & $83.8 \%$ \\
\hline Row 2: age (years) & \multicolumn{2}{|c|}{ Younger than 60 years } & \multicolumn{2}{|l|}{$60-70$ years } & \multicolumn{2}{|l|}{ Older than 70 years } \\
\hline Usual care & 1 & $25.5 \%$ & 1 & $38.0 \%$ & 1 & $26.6 \%$ \\
\hline Center-based & $0.70(0.58-0.84)$ & $74.6 \%$ & $1.06(0.76-1.49)$ & $29.2 \%$ & $0.77(0.34-1.75)$ & $73.4 \%$ \\
\hline Home-based & $0.84(0.45-1.54)$ & $49.9 \%$ & $1.14(0.44-2.99)$ & $28.6 \%$ & & \\
\hline Tele-based & $1.01(0.65-1.55)$ & $27.8 \%$ & $0.20(0.01-4.26)$ & $82.5 \%$ & & \\
\hline Combined & $0.42(0.04-4.62)$ & $72.1 \%$ & $0.57(0.21-1.52)$ & $71.7 \%$ & & \\
\hline Row 3: AMI & \multicolumn{2}{|c|}{ Patients diagnosed with CHD } & \multicolumn{2}{|c|}{ Patients diagnosed with AMI } & & \\
\hline Usual care & 1 & $24.4 \%$ & 1 & $32.6 \%$ & & \\
\hline Center-based & $0.81(0.50-1.33)$ & $55.2 \%$ & $0.75(0.61-0.92)$ & $69.9 \%$ & & \\
\hline Home-based & $0.87(0.48-1.58)$ & $47.4 \%$ & $0.96(0.41-2.25)$ & $41.7 \%$ & & \\
\hline Tele-based & $0.91(0.55-1.49)$ & $41.7 \%$ & $1.21(0.48-3.03)$ & $26.2 \%$ & & \\
\hline Combined & $0.55(0.21-1.45)$ & $81.4 \%$ & $0.26(0.01-5.31)$ & $79.6 \%$ & & \\
\hline Row 4: counseling & \multicolumn{2}{|c|}{ Intervention included counseling } & \multicolumn{2}{|c|}{$\begin{array}{l}\text { Intervention did not include } \\
\text { counseling }\end{array}$} & & \\
\hline Usual care & 1 & $11.0 \%$ & 1 & $21.8 \%$ & & \\
\hline Center-based & $0.73(0.55-0.96)$ & $70.7 \%$ & $0.77(0.61-0.97)$ & $67.7 \%$ & & \\
\hline Home-based & $0.66(0.25-1.77)$ & $68.3 \%$ & $0.91(0.55-1.48)$ & $41.4 \%$ & & \\
\hline Tele-based & & & $0.97(0.61-1.54)$ & $32.2 \%$ & & \\
\hline Combined & & & $0.51(0.20-1.27)$ & $87.0 \%$ & & \\
\hline
\end{tabular}

Effect estimates are presented as the relative risk compared to usual care, and the numbers in parentheses are 95\% confidence intervals. The SUCRA for each intervention was calculated from a cumulative ranking probability that an intervention was above a certain ranking, taking a value between 0 (certainly the worst intervention) and 1 (certainly the best intervention)

SUCRA, surface under the cumulative ranking curve; CHD, coronary heart disease; AMI, acute myocardial infarction

patient domestic or occupational responsibilities, considerable effort has been expended to identify more feasible and costeffective programs. ${ }^{79}$

Previous meta-analyses have demonstrated that homebased CR programs not only significantly reduce the risks of mortality and the incidence of cardiac events among patients with CHD but also exert beneficial effects with respect to functional outcomes, as these programs increase exercise capacity, improve quality of life, and decrease systolic blood pressure. ${ }^{11,80}$ However, our study found that home-based CR is not significantly different from usual care regarding allcause death and CV death. Previous studies have found that home-based CR has a higher incidence of hospital readmission and revascularization than center-based $\mathrm{CR}^{46,81}$ Our analysis demonstrated no difference in rePCI, readmission, and revascularization rates between center-based and home-based CR. It is universally accepted that the availability of home-based programs may enable more patients to participate in exercisebased CR, thereby improving patient uptake and adherence. However, in our study, the program duration of home-based $\mathrm{CR}$ was not longer than that of center-based $\mathrm{CR}$, which indicates that researchers need to test a longer duration to observe patient uptake and adherence.

Furthermore, our detailed analysis of exercise type demonstrated that strength training combined with aerobic training was seen only in center-based CR or combined CR. The materials provided to patients participating in home-based CR exercise programs are usually limited to written instructions, exercise recording logs, and simple counting devices. These methods cannot provide sufficient feedback regarding the quality of the prescribed exercises nor can they be used by clinicians to determine whether patients performed their prescribed exercises correctly or performed the required number of repetitions for each exercise. Our results indicate that more evidence is needed regarding the efficacy of homebased CR programs, which has been recommended as an alternative rehabilitation model in cardiovascular disease prevention by most guidelines.

Since home-based CR lacks exercise guidelines and monitoring, controversy exists regarding whether it is as effective as center-based CR. A new type of CR based on information and communication technology, known as tele-based CR, has recently attracted increasing attention from clinicians. Telebased CR is designed to provide patients with additional feedback, education, and counseling and is supported by sensor technology, communication technology, and data analysis techniques. ${ }^{10}$ Tele-based techniques are increasingly used in $\mathrm{CR}$ and secondary prevention.

Previous systematic reviews indicate that tele-based CR improves cardiovascular risk factors and health-related quality of life, decreases the risks of adverse events, and is costeffective. ${ }^{82}$ Despite the lack of long-term follow-up data regarding tele-based CR, emerging evidence indicates that improvements in tele-monitoring technology have enhanced patient exercise capacity compared with regular center-based training. If performed properly, tele-based CR may be a 
promising alternative means of providing CR for outpatients and ensuring that patients undergo appropriate clinical assessments and receive guidance from providers. ${ }^{12,82,83}$ However, tele-based CR programs are not currently superior to traditional CR with respect to training frequency, exercise duration, training type, or training intensity. Similar to center-based CR exercise intensity, tele-based CR exercise intensity is controlled by physiological indices, with the assistance of telehealth technology. The duration of tele-based CR programs range from 1 month to 1 year, similar to that of traditional CR programs, and may be further improved in future studies. Considering health economics, tele-based CR may be more cost-effective than center-based $\mathrm{CR}$ and be safer and more effective than home-based CR.

As tele-based CR is a young and rapidly evolving field, the innovative technology used in the field lacks a fully developed role in patient care. Moreover, this technology has not demonstrated a sustained ability to motivate patients to undergo tele-based CR. Since data on direct comparisons of the mortality associated with tele-based CR and center-based CR are scarce, the results of our NMA, which demonstrated that the mortality of tele-based CR is similar to that of center-based CR and home-based CR, should be taken seriously. Given that the development of tele-technology is ongoing, the results described herein indicate that tele-based CR should be prescribed with caution.

Similar to other systematic reviews, our study had several limitations. First, few RCTs, with a relatively small number of patients, evaluated tele-based CR or combined CR. Thus, the results of the comparisons involving these $\mathrm{CR}$ programs are imprecise. Second, the methods of the included studies were not well described. Several studies were found to have high risks of bias, although bias did not appear to have affected the outcomes of interest in this review. In some studies, CR involved multidisciplinary rehabilitation rather than just exercise training. The measures other than rehabilitation exercise, such as counseling, a healthier diet, and intermittent oxygen therapy, were often included in the intervention groups but not in the usual care groups and were thus too different to control for. Additionally, most of the studies lacked sufficient information regarding whether randomization was conducted properly or whether allocation concealment was achieved. Third, our searches were limited to English-language publications and published data. Fourth, some of the results of the secondary endpoint analysis may be problematic, as $\mathrm{CABG}$ and $\mathrm{PCI}$ have the potential to temporarily decrease mortality risk. Fifth, advances in medical management post-MI made it difficult to determine the additional benefits of individual treatments. Finally, we did not evaluate differences in cost-effectiveness, functional exercise capacity, or health-related quality of life that were noted among the CR programs.

In conclusion, the present NMA summarized the most comprehensive information regarding several CR methods. Current evidence suggests that center-based CR is the most appropriate therapeutic approach for patients with CHD. As home-based CR and tele-based CR can save time, money, effort, and resources and may be preferred by patients, their efficacy should be investigated further in future studies.

Contributors: Mao Chen had full access to all of the data in the study and takes responsibility for the integrity of the data and the accuracy of the data analysis. Study concept and design: Mao Chen, Fang-yang Huang, and Tian-li Xia. Acquisition, analysis, or interpretation of data: Tian-li Xia, Fang-yang Huang, Yong Peng, Bao-tao Huang, Xiao-bo Pu, Yong Yang, Hua Chai, and Mao Chen. Drafting of the manuscript: Tian-li Xia, Fang-yang Huang, Yong Peng, and Baotao Huang. Critical revision of the manuscript for important intellectual content: Tian-li Xia and Fang-yang Huang. Statistical analysis: Fang-yang Huang. Administrative, technical, or material support: Bao-tao Huang, Xiao-bo Pu, and Yong Yang. Study supervision: Tian-li Xia, Hua Chai, and Mao Chen.

Corresponding Author: Mao Chen, Department of Cardiology, West China Hospital Sichuan University, 37 Guoxue Street, Chengdu 610041, People's Republic of China (e-mail: hmaochen@vip.sina.com).

Funders This research was funded by the National Natural Science Foundation of China (grant number: 81370219 and 81400267. Beijing, China).

\section{Compliance with Ethical Standards:}

Conflict of Interest: The authors declare that they do not have a conflict of interest.

\section{REFERENCES}

1. Anderson L, Oldridge $\mathbf{N}$, Thompson DR, et al. Exercise-based cardiac rehabilitation for coronary heart disease: Cochrane systematic review and meta-analysis. J Am Coll Cardiol. 2016;67:1-12.

2. Giallauria $\mathbf{F}$, Cirillo $\mathbf{P}$, Lucci $\mathbf{R}$, et al. Left ventricular remodelling in patients with moderate systolic dysfunction after myocardial infarction: favourable effects of exercise training and predictive role of $\mathrm{N}$-terminal pro-brain natriuretic peptide. Eur J Cardiov Prev R. 2008;15:115-20.

3. Clark AM, Hartling L, Vandermeer B, McAlister FA. Meta-analysis: secondary prevention programs for patients with coronary artery disease. Ann Intern Med. 2005;143:659-72.

4. Engblom E, Korpilahti K, Hamalainen H, Puukka P, Ronnemaa $\mathbf{T}$ Effects of five years of cardiac rehabilitation after coronary artery bypass grafting on coronary risk factors. Am J Cardiol. 1996;78:1428-31.

5. Piepoli MF, Hoes AW, Agewall S, et al. 2016 European guidelines on cardiovascular disease prevention in clinical practice. Eur Heart J. 2016;37:2315-81.

6. Carlsson R, Lindberg G, Westin L, Israelsson B. Influence of coronary nursing management follow up on lifestyle after acute myocardial infarction. Heart. 1997;77:256-9.

7. Martinez DG, Nicolau JC, Lage RL, et al. Effects of long-term exercise training on autonomic control in myocardial infarction patients. Hypertension. 2011;58:1049-56.

8. Debusk RF, Haskell WL, Miller NH, et al. Medically directed at-home rehabilitation soon after clinically uncomplicated acute myocardialinfarction - a new model for patient-care. Am J Cardiol. 1985;55:251-7.

9. Miller NH, Haskell WL, Berra $\mathbf{K}$, Debusk RF. Home versus group exercise training for increasing functional-capacity after myocardialinfarction. Circulation. 1984;70:645-9.

10. Patel S, Park $\mathbf{H}$, Bonato $\mathbf{P}$, Chan $\mathbf{L}$, Rodgers $\mathbf{M}$. A review of wearable sensors and systems with application in rehabilitation. J Neuroeng Rehabil. 2012;9:21.

11. Dalal HM, Zawada A, Jolly K, Moxham T, Taylor RS. Home based versus centre based cardiac rehabilitation: cochrane systematic review and meta-analysis. BMJ. 2010;340:b5631.

12. Rawstorn JC, Gant N, Direito A, Beckmann C, Maddison R. Telehealth exercise-based cardiac rehabilitation: a systematic review and metaanalysis. Heart. 2016;102:1183-92. 
13. Liberati A, Altman DG, Tetzlaff $\mathbf{J}$, et al. The PRISMA statement for reporting systematic reviews and meta-analyses of studies that evaluate healthcare interventions: explanation and elaboration. BMJ. 2009;339:b2700.

14. Harris RJ, Bradburn MJ, Deeks JJ, Harbord RM, Altman DG, Sterne J. Metan: fixed- and random-effects meta-analysis. Stata J. 2008;8:3-28.

15. Higgins JP, Thompson SG. Quantifying heterogeneity in a metaanalysis. Stat Med. 2002;21:1539-58.

16. Jackson D, Barrett JK, Rice S, White IR, Higgins JP. A design-bytreatment interaction model for network meta-analysis with random inconsistency effects. Stat Med. 2014;33:3639-54.

17. Chaimani A, Salanti G. Visualizing assumptions and results in network meta-analysis: the network graphs package. Stata J. 2015; 15:905-50.

18. Dias S, Welton NJ, Caldwell DM, Ades AE. Checking consistency in mixed treatment comparison meta-analysis. Stat Med. 2010;29:932-44.

19. Chaimani A, Higgins JPT, Mavridis D, Spyridonos P, Salanti G. Graphical tools for network meta-analysis in STATA. PLoS One. 2013;8:e76654.

20. Salanti G, Ades AE, Ioannidis JPA. Graphical methods and numerical summaries for presenting results from multiple-treatment meta-analysis: an overview and tutorial. J Clin Epidemiol. 2011;64:163-71.

21. World Health Organization, Regional Office for Europe. Rehabilitation and comprehensive secondary prevention after acute myocardial infarction: report on a study. Copenhagen: World Health Organization, WHO Publications Centre USA; 1983.

22. Aronov DM. [The problem of duration of hospital stay of patients with acute myocardial infarction]. Kardiologiia. 2010;50:79-85.

23. Belardinelli R, Paolini I, Cianci G, Piva R, Georgiou D, Purcaro A. Exercise training intervention after coronary angioplasty: the ETICA trial. J Am Coll Cardiol. 2001;37:1891-900.

24. Campbell NC, Thain J, Deans HG, Ritchie LD, Rawles JM, Squair JL. Secondary prevention clinics for coronary heart disease: randomised trial of effect on health. BMJ. 1998;316:1434-7.

25. Bethell HJN, Mullee MA. A controlled trial of community based coronary rehabilitation. Brit Heart J. 1990;64:370-5.

26. Bengtsson K. Rehabilitation after myocardial infarction. A controlled study. Scand J Rehabil Med. 1983;15:1.

27. Bertie J, King A, Reed N, Marshall A, Ricketts C. Benefits and weaknesses of a cardiac rehabilitation programme. J Roy Coll Phys Lond. 1992;26:147-51.

28. Briffa TG, Eckermann SD, Griffiths AD, et al. Cost-effectiveness of rehabilitation after an acute coronary event: a randomised controlled trial. Med J Aust. 2005; 183:450-5.

29. Carlsson R. Serum cholesterol, lifestyle, working capacity and quality of life in patients with coronary artery disease. Experiences from a hospitalbased secondary prevention programme. Scand Cardiovasc J Suppl. 1998;50:1-20

30. Carson $\mathbf{P}$, Phillips R, Lloyd $\mathbf{M}$, et al. Exercise after myocardial infarction: a controlled trial. J Roy Coll Phys Lond. 1982;16:147-51.

31. Vale M, Jelinek M, Best J, Santamaria J. Coaching patients with coronary heart disease to achieve the target cholesterol: a method to bridge the gap between evidence-based medicine and the "real world"-randomized controlled trial. J Clin Epidemiol. 2002;55:245-52.

32. Vale MJ, Jelinek MV, Best JD, et al. Coaching patients on achieving cardiovascular health (coach): a multicenter randomized trial in patients with coronary heart disease. Arch Intern Med. 2003;163:2775-83.

33. Dalal HM, Evans PH, Campbell JL, et al. Home-based versus hospitalbased rehabilitation after myocardial infarction: a randomized trial with preference arms-Cornwall Heart Attack Rehabilitation Management Study (CHARMS). Int J Cardiol. 2007;119:202-11.

34. DeBusk RF, Miller NH, Superko HR, et al. A case-management system for coronary risk factor modification after acute myocardial infarction. Ann Intern Med. 1994;120:721-9.

35. Dugmore LD, Tipson RJ, Phillips MH, et al. Changes in cardiorespiratory fitness, psychological wellbeing, quality of life, and vocational status following a 12 month cardiac exercise rehabilitation programme. Heart. 1999;81:359-66.

36. Erdman RAM, Duivenvoorden HJ, Verhage $\mathbf{F}$, Kazemier M Hugenholtz PG. Predictability of beneficial effects in cardiac rehabilitation: a randomized clinical trial of psychosocial variables. J Cardiopulm Rehabil. 1986;6:206-13.

37. Fletcher BJ, Dunbar SB, Felner JM, et al. Exercise testing and training in physically disabled men with clinical-evidence of coronary-artery disease. Am J Cardiol. 1994;73:170-4.
38. Fridlund B, Hogstedt B, Lidell E, Larsson PA. Recovery after myocardial infarction. Effects of a caring rehabilitation programme. Scand J Caring Sci. 1991;5:23-32.

39. Gordon NF, English CD, Contractor AS, et al. Effectiveness of three models for comprehensive cardiovascular disease risk reduction. Am J Cardiol. 2002;89:1263-8.

40. Giallauria F, Lucci R, D'Agostino M, et al. Two-year multicomprehensive secondary prevention program: favorable effects on cardiovascular functional capacity and coronary risk profile after acute myocardial infarction. J Cardiovasc Med. 2009;10:772-80.

41. Haskell WL, Alderman EL, Fair JM, et al. Effects of intensive multiple risk factor reduction on coronary atherosclerosis and clinical cardiac events in men and women with coronary artery disease. The Stanford Coronary Risk Intervention Project (SCRIP). Circulation. 1994;89:975-90.

42. Heller RF, Knapp JC, Valenti LA, Dobson AJ. Secondary prevention after acute myocardial-infarction. Am J Cardiol. 1993;72:759-62.

43. Higgins HC, Hayes RL, McKenna KT. Rehabilitation outcomes following percutaneous coronary interventions (PCI). Patient Educ Couns. 2001;43:219-30.

44. Hofman-Bang $\mathbf{C}$, Lisspers $\mathbf{J}$, Nordlander $\mathbf{R}$, et al. Two-year results of a controlled study of residential rehabilitation for patients treated with percutaneous transluminal coronary angioplasty - a randomized study of a multifactorial programme. Eur Heart J. 1999;20: 1465-74.

45. Houle J, Doyon O, Vadeboncoeur N, Turbide G, Diaz A, Poirier P. Effectiveness of a pedometer-based program using a socio-cognitive intervention on physical activity and quality of life in a setting of cardiac rehabilitation. Can J Cardiol. 2012;28:27-32

46. Jolly K, Taylor R, Lip G, Greenfield S, Raftery J. The Birmingham Rehabilitation Uptake Maximisation Study (BRUM). Home-based compared with hospital-based cardiac rehabilitation in a multi-ethnic population: cost-effectiveness and patient adherence. Health Technol Assess. 2007;11:118.

47. Jones DA, West RR. Psychological rehabilitation after myocardial infarction: multicentre randomised controlled trial. BMJ. 1996;313:1517-21

48. Kallio V, Hamalainen H, Hakkila J, Luurila OJ. Reduction in sudden deaths by a multifactorial intervention program after acute myocardialinfarction. Lancet. 1979;2:1091-4.

49. Kovoor P, Lee AK, Carrozzi F, et al. Return to full normal activities including work at two weeks after acute myocardial infarction. Am J Cardiol. 2006;97:952-8.

50. La Rovere MT, Bersano C, Gnemmi M, Specchia G, Schwartz PJ. Exercise-induced increase in baroreflex sensitivity predicts improved prognosis after myocardial infarction. Circulation. 2002;106:945-9.

51. Lear SA, Ignaszewski A, Linden W, et al. The extensive lifestyle management intervention (ELMI) following cardiac rehabilitation trial. Eur Heart J. 2003;24:1920-7.

52. Lewin B, Robertson IH, Cay EL, Irving JB, Campbell M. Effects of selfhelp post-myocardial-infarction rehabilitation on psychological adjustment and use of health-services. Lancet. 1992;339:1036-40.

53. Maddison R, Pfaeffli L, Whittaker R, et al. A mobile phone intervention increases physical activity in people with cardiovascular disease: results from the HEART randomized controlled trial. Eur J Prev Cardiol. 2015;22:701-9.

54. Manchanda S, Narang $\mathbf{R}$, Reddy $\mathbf{K}$, et al. Retardation of coronary atherosclerosis with yoga lifestyle intervention. J Assoc Physicians India. 2000;48:687-94.

55. Maroto Montero JM, Artigao Ramirez R, Morales Duran MD, de Pablo Zarzosa C, Abraira V. [Cardiac rehabilitation in patients with myocardial infarction: a 10-year follow-up study]. Rev Esp Cardiol. 2005;58:1181-7.

56. Munk PS, Breland UM, Aukrust P, Ueland T, Kvaloy JT, Larsen AI. High intensity interval training reduces systemic inflammation in postPCI patients. Eur J Cardiovasc Prev Rehabil. 2011;18:850-7.

57. Mutwalli HA, Fallows SJ, Arnous AA, Zamzami MS. Randomized controlled evaluation shows the effectiveness of a home-based cardiac rehabilitation program. Saudi Med J. 2012;33:152-9.

58. Niebauer $\mathbf{J}$, Hambrecht $\mathbf{R}$, Velich $\mathbf{T}$, et al. Attenuated progression of coronary artery disease after 6 years of multifactorial risk intervention: role of physical exercise. Circulation. 1997;96:2534-41.

59. Oerkild B, Frederiksen M, Hansen JF, Prescott E. Home-based cardiac rehabilitation is an attractive alternative to no cardiac rehabilitation for elderly patients with coronary heart disease: results from a randomised clinical trial. BMJ Open. 2012;2:e001820. 
60. Oldridge N, Guyatt G, Jones $\mathbf{N}$, et al. Effects on quality of life with comprehensive rehabilitation after acute myocardial infarction. Am J Cardiol. 1991;67:1084-9.

61. Ornish D, Scherwitz LW, Billings $\mathbf{J H}$, et al. Intensive lifestyle changes for reversal of coronary heart disease. JAMA. 1998;280:2001-7.

62. Reid RD, Morrin LI, Beaton LJ, et al. Randomized trial of an internetbased computer-tailored expert system for physical activity in patients with heart disease. Eur J Prev Cardiol. 2012;19:1357-64.

63. Roman O, Gutierrez M, Luksic I, et al. Cardiac rehabilitation after acute myocardial infarction. 9-year controlled follow-up study. Cardiology. 1983;70:223-31.

64. Schuler G, Hambrecht R, Schlierf G, et al. Regular physical exercise and low-fat diet. Effects on progression of coronary artery disease. Circulation. 1992;86:1-11.

65. Seki E, Watanabe Y, Shimada $\mathbf{K}$, et al. Effects of a phase III cardiac rehabilitation program on physical status and lipid profiles in elderly patients with coronary artery disease - Juntendo Cardiac Rehabilitation Program (J-CARP). Circ J. 2008;72:1230-4.

66. Shaw LW. Effects of a prescribed supervised exercise program on mortality and cardiovascular morbidity in patients after myocardial infarction. The National Exercise and Heart Disease Project. Am J Cardiol. 1981;48:39-46.

67. Sivarajan ES, Bruce RA, Lindskog BD, Almes MJ, Belanger L, Green B. Treadmill test responses to an early exercise program after myocardial infarction: a randomized study. Circulation. 1982;65:1420-8.

68. Specchia G, De Servi S, Scire` A, et al. Interaction between exercise training and ejection fraction in predicting prognosis after a first myocardial infarction. Circulation. 1996;94:978-82.

69. Stern MJ, Gorman PA, Kaslow L. The group counseling v exercise therapy study. A controlled intervention with subjects following myocardial infarction. Arch Intern Med. 1983;143:1719-25.

70. Toobert DJ, Glasgow RE, Radcliffe JL. Physiologic and related behavioral outcomes from the Women's Lifestyle Heart Trial. Ann Behav Med. 2000;22:1-9.

71. Vermeulen A, Lie KI, Durrer D. Effects of cardiac rehabilitation after myocardial infarction: changes in coronary risk factors and long-term prognosis. Am Heart J. 1983;105:798-801.

72. Vestfold Heartcare Study Group. Influence on lifestyle measures and fiveyear coronary risk by a comprehensive lifestyle intervention programme in patients with coronary heart disease. Eur J Cardiovasc Prev Rehabil. 2003; 10:429-37.
73. Wang W, Chair SY, Thompson DR, Twinn SF. Effects of home-based rehabilitation on health-related quality of life and psychological status in Chinese patients recovering from acute myocardial infarction. Heart Lung. 2012;41:15-25.

74. West RR, Jones DA, Henderson AH. Rehabilitation after myocardial infarction trial (RAMIT): multi-centre randomised controlled trial of comprehensive cardiac rehabilitation in patients following acute myocardial infarction. Heart. 2012;98:637-44

75. Wilhelmsen L, Sanne H, Elmfeldt D, Grimby G, Tibblin G, Wedel H. A controlled trial of physical training after myocardial infarction. Effects on risk factors, nonfatal reinfarction, and death. Prev Med. 1975;4:491-508.

76. Yu CM, Lau CP, Chan J, et al. A short course of cardiac rehabilitation program is highly cost effective in improving long-term quality of life in patients with recent myocardial infarction or percutaneous coronary intervention. Arch Phys Med Rehab. 2004;85:1915-22.

77. Young W, Rewa G, Goodman SG, et al. Evaluation of a community-based inner-city disease management program for postmyocardial infarction patients: a randomized controlled trial. Can Med Assoc J. 2003;169:905-10.

78. Zwisler, Abedini S. Hospital-based comprehensive cardiac rehabilitation versus usual care among patients with congestive heart failure, ischemic heart disease, or high risk of ischernic heart disease: 12-month results of a randomized clinical trial (vol 155, pg 1106, 2008). Am Heart J. 2008;156:276-.

79. Frasure-Smith N, Lesperance F, Prince RH, et al. Randomised trial of home-based psychosocial nursing intervention for patients recovering from myocardial infarction. Lancet. 1997;350:473-9.

80. Jolly K, Taylor RS, Lip GY, Stevens A. Home-based cardiac rehabilitation compared with centre-based rehabilitation and usual care: a systematic review and meta-analysis. Int J Cardiol. 2006;111:343-51.

81. Taylor RS, Watt A, Dalal HM, et al. Home-based cardiac rehabilitation versus hospital-based rehabilitation: a cost effectiveness analysis. Int $\mathrm{J}$ Cardiol. 2007;119:196-201.

82. Neubeck L, Redfern J, Fernandez R, Briffa T, Bauman A, Freedman SB. Telehealth interventions for the secondary prevention of coronary heart disease: a systematic review. Eur J Cardiovasc Prev Rehabil. 2009;16:281-9.

83. Frederix I, Van Driessche N, Hansen D, et al. Increasing the mediumterm clinical benefits of hospital-based cardiac rehabilitation by physical activity telemonitoring in coronary artery disease patients. Eur J Prev Cardiol. 2015;22:150-8. 